\title{
Shape-Dependent Interactions of Manganese Oxide Nanomaterials with Lipid Bilayer Vesicles
}

\author{
Langmuir
}

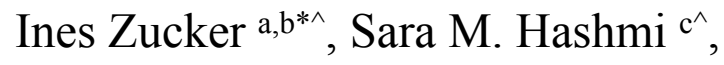

${ }^{\wedge}$ Co-first authors

Jason Yang ${ }^{\mathrm{d}}$, Yulian $\mathrm{He}^{\mathrm{d}}{ }^{\text {, Lisa D. Pfefferle }}{ }^{\mathrm{d}}$, and Menachem Elimelech ${ }^{\mathrm{d}}$

a School of Mechanical Engineering, Faculty of Engineering, Tel Aviv University, Tel Aviv 69978, Israel

b Porter School of Environmental Studies, Faculty of Exact Sciences, Tel Aviv University, Tel Aviv 69978, Israel

c States Department of Chemical Engineering, Northeastern University, Boston, MA 021155000, United

d Department of Chemical and Environmental Engineering, Yale University, New Haven, Connecticut 06520-8286, United States 

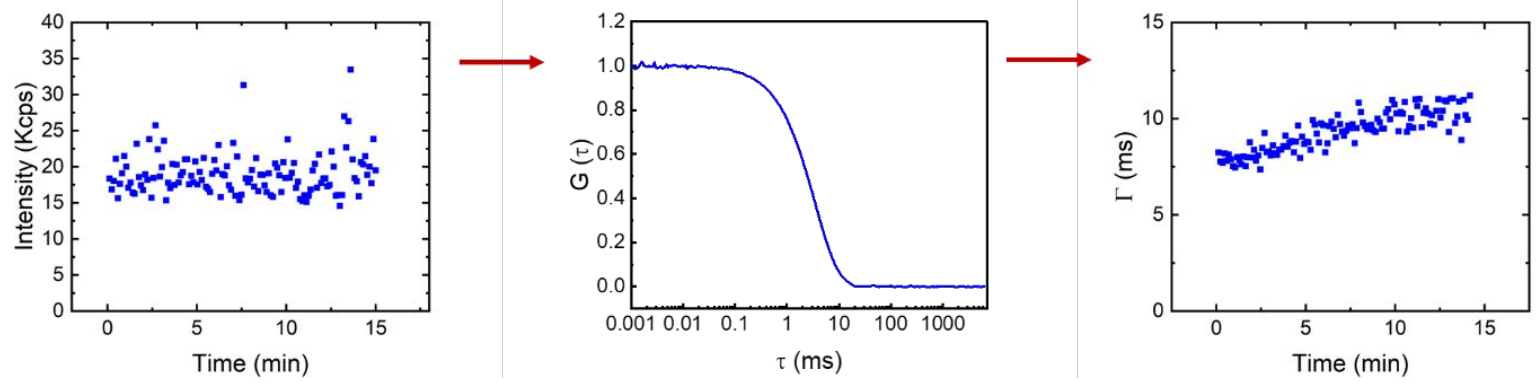

Figure S1. Dynamic light scattering data processing procedure. Light scattering instrument measures fluctuations in scattered light that arise from Brownian diffusive motion (left) and calculates the intensity correlation function (center). Finally, a series of correlation functions collected over time are each fitted to a decaying exponential $(\Gamma)$ to obtain diffuse time scale(s). 


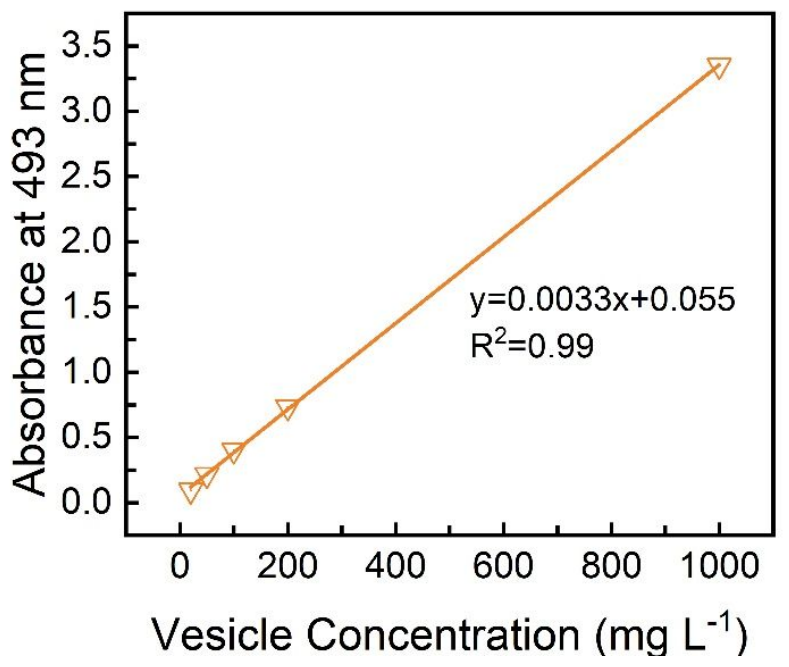

Figure S2. Linear calibration of vesicle concentration to absorbance at $493 \mathrm{~nm}$. 


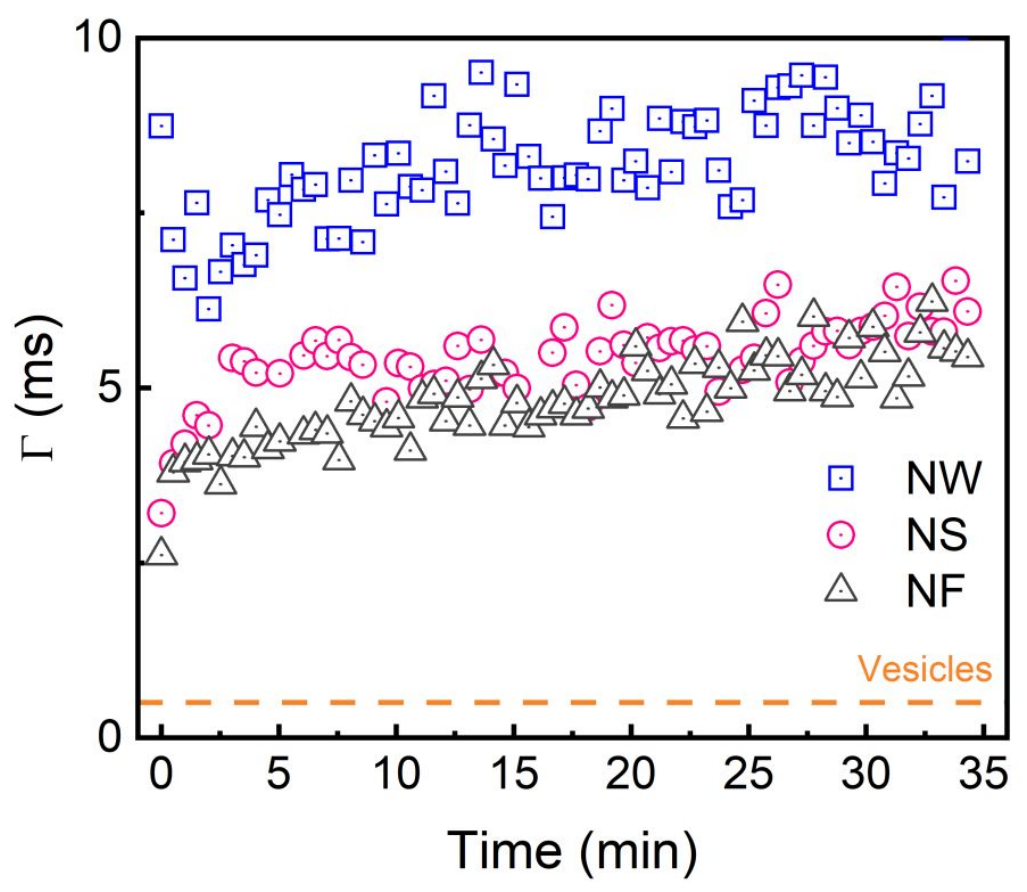

Figure S3. Control measurements of aggregation kinetics for $20 \mathrm{mg} \mathrm{L}^{-1}$ manganese oxide $\left(\mathrm{MnO}_{2}\right)$ nanowires (NW), nanosheets (NS), and nanoflowers (NF) and lipid vesicles (100 mg L-1) as determined by dynamic light scattering (DLS) cumulate analysis. Light scattering data are presented as $\Gamma$ (diffusive time). 

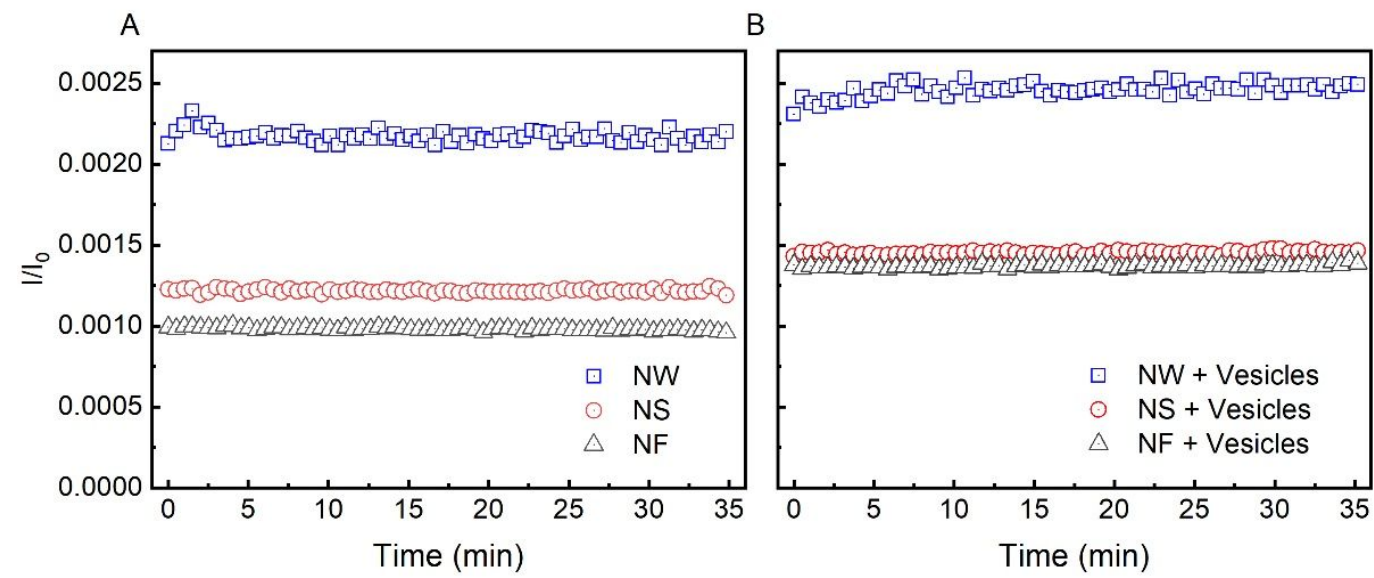

Figure S4. Stable aggregation kinetics for (A) $20 \mathrm{mg} \mathrm{L}^{-1}$ manganese oxide $\left(\mathrm{MnO}_{2}\right)$ nanowires (NW), nanosheets (NS), and nanoflowers (NF) in suspension and (B) interacted with lipid vesicles (100 $\left.\mathrm{mg} \mathrm{L}^{-1}\right)$ ) as determined by dynamic light scattering (DLS). Vertical axis in the ratio of intensity at the detector $(\mathrm{I})$ to the incident intensity $\left(\mathrm{I}_{0}\right)$. Constant intensity demonstrates lack of settling. 


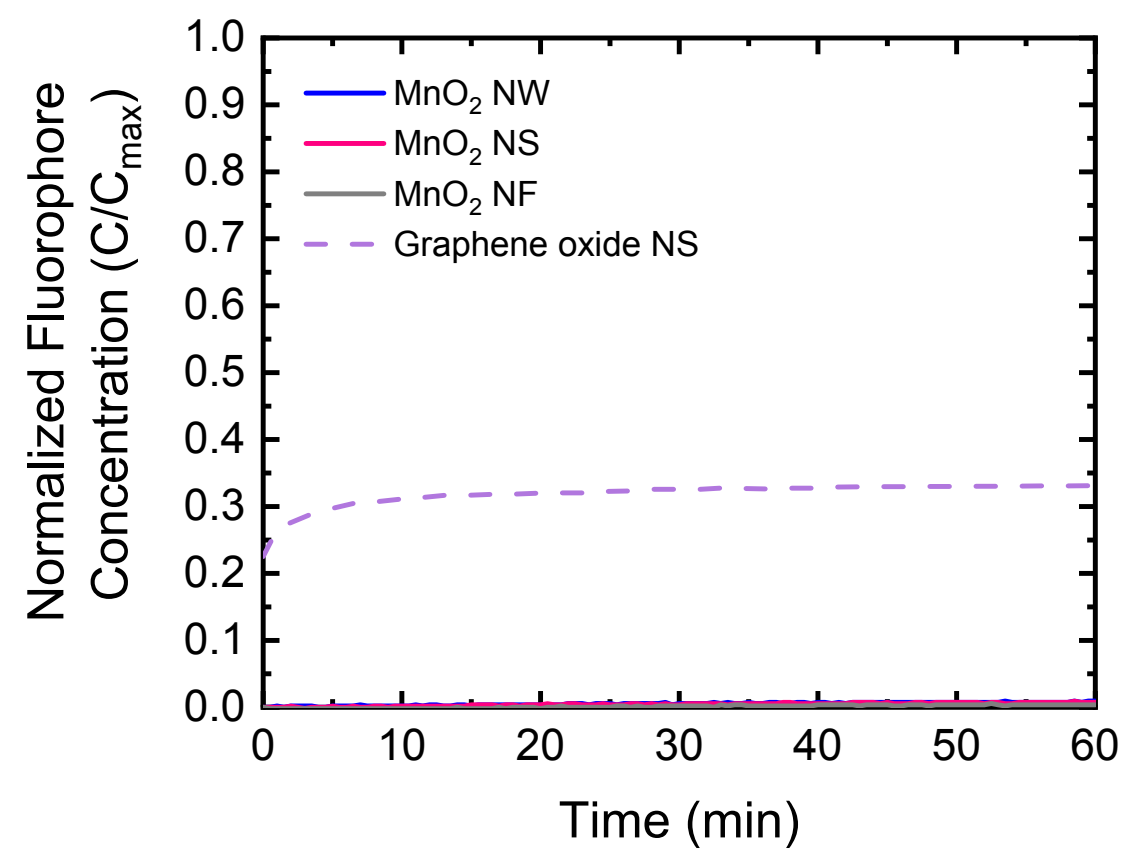

Figure S5. Fluorescence assay of vesicles $\left(100 \mathrm{mg} \mathrm{L}^{-1}\right)$ interacted with $\mathrm{MnO}_{2}$ nanomaterials of different shapes including nanowires (NW), nanosheets (NS), and nanoflowers (NF), as well as and graphene oxide nanosheets $\left(20 \mathrm{mg} \mathrm{L}^{-1}\right)$ showing the fractional dye leakage over time as observed in our former study. ${ }^{1} \mathrm{GO}$ nanosheets were received as an aqueous suspension $(6.2 \mathrm{~g} / \mathrm{L})$ from a commercial source (Graphene Supermarket, NY, US). $\mathrm{MnO}_{2}$ nanomaterials did not induce loss of vesicle membrane integrity. 
A

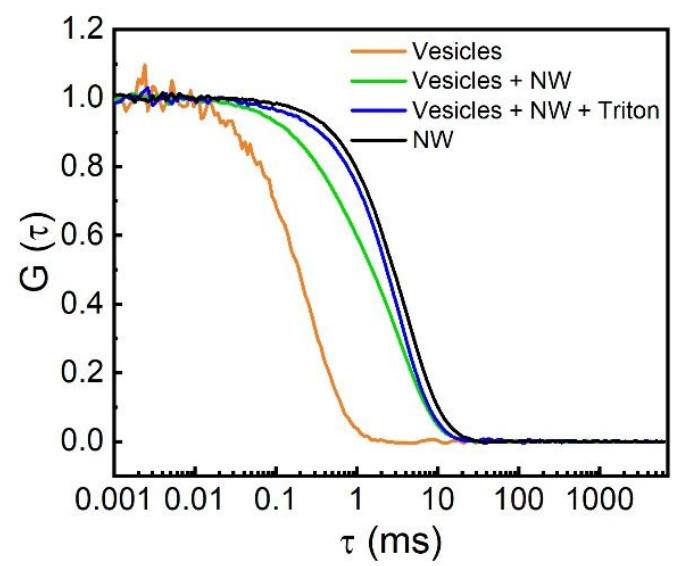

B

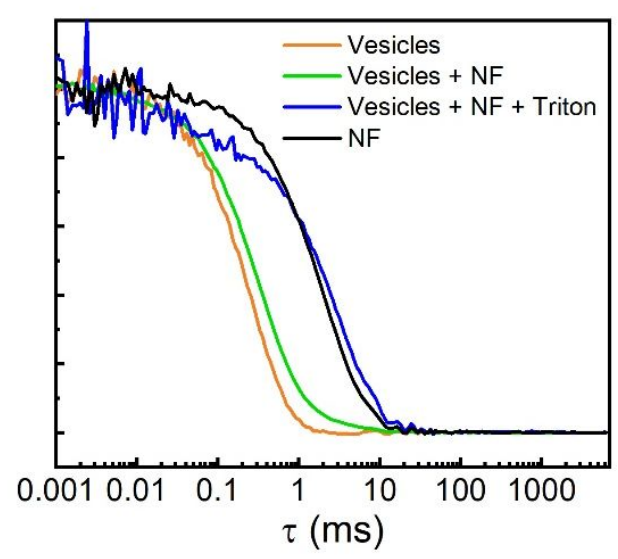

Figure S6. Intensity correlation function of $20 \mathrm{mg} \mathrm{L}^{-1} \mathrm{MnO}_{2}$ nanowires (NW) and nanoflowers (NF) interacted with vesicles $\left(100 \mathrm{mg} \mathrm{L}^{-1}\right)$ showing residual broken nanomaterial aggregates and recovery of the control $\mathrm{MnO}_{2}$ nanomaterial diffusive timescale after vesicles were solubilized by Triton X-100. 


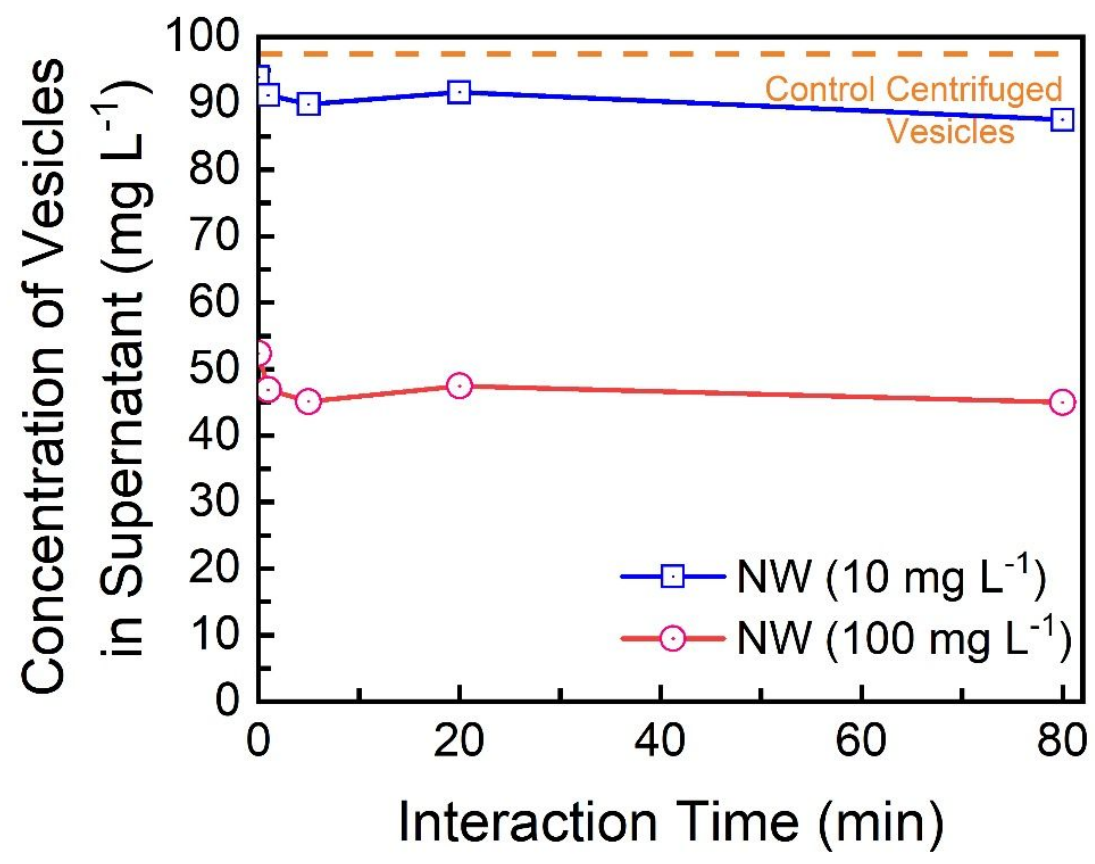

Figure S7. Adsorption isotherms for $100 \mathrm{mg} \mathrm{L}^{-1}$ vesicles interacted with two different concentrations of NWs $\left(10,100 \mathrm{mg} \mathrm{L}^{-1}\right)$ after interaction times of $0,1,5,20$, and 80 mins. Concentration of vesicles in the supernatant is measured after centrifuging for five minutes at $13 \mathrm{k}$ rpm. Vesicle adsorption to NWs reaches equilibrium after one minute of interaction, as the concentration of vesicles measured in the supernatant stabilizes after one minute. Vesicle concentration is calculated from a linear calibration of absorbance at $493 \mathrm{~nm}$ (Figure S2 in the Supporting Information). 


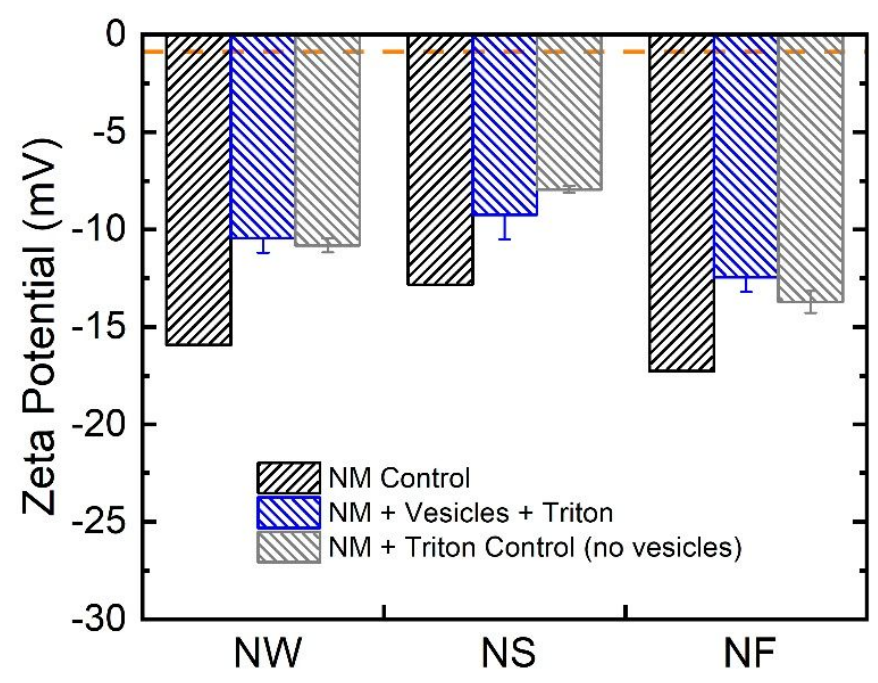

Figure S8. Changes in magnitude of zeta potential of $\mathrm{MnO}_{2}$ with addition of $100 \mathrm{mg} \mathrm{L}^{-1}$ vesicles that are solubilized by a surfactant, Triton X-100. NM Control zeta potentials (black bars) and NM + Triton Control zeta potentials (gray bars) are shown as references, indicating the limited effect of solubilized vesicles on zeta potential. Vesicle zeta potential is shown as an orange dash line. 


\section{References:}

1 I. Zucker, J. R. Werber, Z. S. Fishman, S. M. Hashmi, U. R. Gabinet, X. Lu, C. O. Osuji, L. D. Pfefferle and M. Elimelech, Environ. Sci. Technol. Lett., 2017, 404-409. 